\title{
Stable Isotope Dilution Analysis of Galactitol in Amniotic Fluid: an Accurate Approach to the Prenatal Diagnosis of Galactosemia
}

\author{
CORNELIS JAKOBS, ${ }^{(16)}$ THOMAS G. WARNER, LAWRENCE SWEETMAN, AND \\ WILLIAM L. NYHAN \\ Departments of Pediatrics [C.J.,L.S., W.L.N.] and Neurosciences [T.G.W.], University of California, San Diego, \\ La Jolla, California, USA
}

\begin{abstract}
Summary
A stable isotope dilution assay for galactitol in amniotic fluid has been developed using selected ion monitoring chemical ionization gas chromatography-mass spectrometry of the hexaacetate derivative. $\left[1,1-{ }^{2} \mathrm{H}_{2}\right]$ Galactitol was synthesized for use as the internal standard. Galactitol is a component of normal amniotic fluid with a mean concentration of $0.70 \pm 0.18 \mu \mathrm{mol} / \mathrm{liter}(n=$ 5). The amniotic fluid of a fetus with galactosemia had a concentration of $7.96 \mu \mathrm{mol} / \mathrm{liter}$. Mannitol, sorbitol, and inositol were also found to be normal constituents of amniotic fluid. This stable isotope dilution assay is a rapid accurate method for measurement of galactitol in amniotic fluid for prenatal diagnosis of galactosemia.
\end{abstract}

\section{Abbreviations}

GCMS, gas chromatography-mass spectroscopy

SIM, selected ion monitoring

$\mathrm{D}_{2},{ }^{2} \mathbf{H}_{2}$ label

CI, chemical ionization

Genetically determined disorders of galactose metabolism include classical galactosemia, galactose-1-phosphate uridylyltransferase (EC 2.7.7.12) deficiency, galactokinase (EC 2.7.1.6) deficiency, and uridine diphosphate galactose-4-epimerase (EC 5.1.3.2) deficiency $(6,7,12)$. Each of the enzymes is known to be present in cultured skin fibroblasts and in cultured amniotic fluid cells. The disorders of galactose metabolism have serious implications for the untreated affected child. Galactosemia may lead to death in early infancy, usually from sepsis, and this could occur even in infants detected in programs of routine neonatal screening. Further manifestations are failure to thrive, jaundice, and hepatic cirrhosis. Later, cataracts and mental retardation develop. Patients with galactokinase deficiency develop cataracts. If detection is early, postnatal dietary treatment is effective although there may be some irreversible damage, especially to the central nervous system. Prenatal diagnosis can assist in determining the need for restrictive dietary management during gestation $(11,13)$. Prenatal diagnosis has been reported using enzymatic assays $(5,10)$.

The accumulation of galactitol in body fluids of patients with disorders of galactose metabolism raises the possibility of the development of a rapid, precise chemical method of prenatal diagnosis by direct analysis of the aminiotic fluid for galactitol. Allen et al. (1) have reported the accumulation of galactitol in amniotic fluid and have published (2) a method for the prenatal diagnosis of galactosemia using GC for the determination of galactitol in amniotic fluid. There are two major disadvantages of this method. The first was the use of mannitol as an internal standard, because this compound is a normal constituent of amniotic fluid. Furthermore, the use of GC instrumentation alone has a limited resolving capacity and the detection system is relatively nonspecific, allowing the possible interference by a coeluting compound.

We have developed a stable isotope dilution method for galactitol, utilizing an on-line GCMS system operated in the $\mathrm{CI}$ SIM mode. CI-SIM-GCMS circumvents the limitations of previous methodology and provides stable isotopically labeled [1,1${ }^{2} \mathrm{H}_{2}$ ] galactitol as an internal standard not found in physiological fluids.

\section{MATERIALS AND METHODS}

Normal amniotic fluids were obtained between 16 and 18 weeks of pregnancy by amniocentesis for analysis of chromosomes and stored at $-20^{\circ} \mathrm{C}$ until analyzed. The fluid from a pregnancy with a fetus with galactosemia as indicated by a deficiency of galactose-1-phosphate uridylyltransferase was sent for analysis as sterile fluid frozen in dry ice and stored prior to analysis at $-20^{\circ} \mathrm{C}$. D-Galactitol, D-mannitol, D-sorbitol, and myoinositol were obtained from Sigma, St. Louis, MO. Sulfosalicylic acid was from Aldrich, Milwaukee, WI.

Synthesis of $\left[1,1-2^{2} \mathrm{H}_{2}\right]$ Galactitol. For synthesis of the internal standard, $50 \mathrm{mg}$ of $\left[6,6-{ }^{2} \mathrm{H}_{2}\right]$ galactose, $98 \%$ isotopic purity (MSD Isotopes, Pointe Claire-Dorval, Quebec, Canada) was suspended in $0.5 \mathrm{ml}$ of $50 \mathrm{mmol} /$ liter $\mathrm{NaOH}$ containing $50 \mathrm{mg} \mathrm{NaBH}$. The reaction was allowed to proceed with agitation at room temperature for $4 \mathrm{hr}$. The reaction was terminated by the addition of acetic acid ( $2 \mathrm{~mol} /$ liters), and the mixture was applied to a column $(1.5 \mathrm{~cm}$ ID $\times 2.0 \mathrm{~cm})$ containing Dowex $50\left(\mathrm{H}^{+}\right.$form, Bio-Rad Laboratories, Richmond, CA) with a cover layer of the borate-specific resin IRA-743 $(1.5 \times 6.5 \mathrm{~cm}$, Sigma, St. Louis, $\mathrm{MO}$ ). The column was subsequently washed with $50 \mathrm{ml}$ of water, the eluate was collected, and the solvent was removed under vacuum. The residue was resuspended in water and purified further by gel chromatography on a Bio-Gel P-2 column $(1.0 \mathrm{~cm}$ ID $\times 90 \mathrm{~cm}$, Bio-Rad Laboratories, Richmond, Ca), collecting 1.0-ml fractions. Each fraction was monitored for carbohydrate by removing an aliquot and testing for charring with $50 \% \mathrm{H}_{2} \mathrm{SO}_{4}$ at $100^{\circ} \mathrm{C}$ for $10 \mathrm{~min}$. The fractions containing the reduced product were pooled and the water was removed under vacuum. The residue was suspended in water, filtered through a $0.2-\mu \mathrm{m}$ filter, and lyophilized. The yield was $39.6 \mathrm{mg}$ of $\mathrm{D}_{2}$-galactitol or about $80 \%$.

The chemical and isotopic purities of the purified $\left[1,1-{ }^{2} \mathrm{H}_{2}\right]$ galactitol were compared to those of D-galactitol and the $[6,6-$ 
${ }^{2} \mathrm{H}_{2}$ ]galactose starting material. One micromole of each compound was acetylated in duplicate as described below for the preparation of amniotic fluid samples and $50 \mathrm{nmol}$ were injected into the GCMS. The SP-2340 column was programmed from 200 to $275^{\circ} \mathrm{C}$ at $8^{\circ} \mathrm{C} / \mathrm{min}$ with $20 \mathrm{ml} / \mathrm{min}$ of helium as the carrier gas and with ammonia as the reagent gas at a pressure of 9 pascals in the source. CI spectra from $\mathrm{m} / z 50$ to 650 were obtained every $2 \mathrm{sec}$ and peak areas were determined from the total ion chromatograms. After subtracting the reagent and solvent blank peaks, the two peaks corresponding to the hexaacetate and pentaacetate derivatives constituted $99.53 \%$ of the peak areas for galactitol and $99.52 \%$ of the peak areas for $\mathrm{D}_{2}$-galactitol. The total of the peak areas of the two derivatives for $D_{2}$-galactitol were $100.45 \%$ of those of galactitol, indicating identical purities. The peak area of the hexaacetate was $90.5 \%$ of the combined peak areas of the hexaacetate and pentaacetate for galactitol and $91.8 \%$ for $\mathrm{D}_{2}$-galactitol, indicating similar derivatizations. The relative intensities of the isotope cluster of the ammonium adduct pseudomolecular ion in the CI spectrum of galactitol hexaacetate were $18.71 \%$ for $\mathrm{m} / z 453 / 452$ and $4.61 \%$ for $\mathrm{m} / z 454 / 452$. These were similar to the theoretically expected relative intensities of 20.78 and $4.46 \%$, indicating minimal hydrogen losses. The corresponding relative intensities for the $\mathrm{CI}$ spectrum of $\mathrm{D}_{2}$ galactitol hexaacetate were $18.63 \%$ for $\mathrm{m} / \mathrm{z} 455 / 454$ and $4.47 \%$ for $m / z 456 / 454$. The close similarities to the relative abundances for galactitol hexaacetate showed that there were no significant isotope effects of the deuterium. The isotopic purity of the $\mathrm{D}_{2^{-}}$ galactitol hexaacetate calculated from the intensities at $\mathrm{m} / z 452$, 453 , and 454 was $99.84 \%$ with two deuteriums, $0 \%$ with one deuterium, and $0.16 \%$ with no deuteriums. This corresponded closely to the isotopic purity of the pentaacetate of the $\mathrm{D}_{2}$ galactose starting material determined similarly to be $99.67 \%$ with two deuteriums, $0 \%$ with one deuterium, and $0.33 \%$ with no deuteriums.

Preparation of amniotic fluid samples. The procedure of Allen et al. (2) was modified. To $1 \mathrm{ml}$ of amniotic fluid, $5 \mathrm{nmol}$ of $\mathrm{D}_{2^{-}}$ galactitol were added and proteins were precipitated by the addition of $200 \mu \mathrm{l}$ of $4 \%$ sulfosalicylic acid. Following centrifugation, the supernatant amniotic fluid was transferred to a Pasteur pipette filled with $4 \mathrm{~cm}$ of Dowex AG 2-X8, 200-400 mesh $\left(\mathrm{OH}^{-}\right.$form, freshly prepared) at neutral $\mathrm{pH}$ with a top layer of 2 cm of Dowex AG 50W-X4, 100-200 mesh $\left(\mathrm{H}^{+}\right.$form) at neutral $\mathrm{pH}$. The column was eluted with $6 \mathrm{ml}$ of deionized, distilled water, and the eluate was lyophilized. To the dried residue was added $200 \mu \mathrm{l}$ of pyridine and $200 \mu \mathrm{l}$ of acetic anhydride, and the sample was heated for $60 \mathrm{~min}$ at $80^{\circ} \mathrm{C}$ for form the hexaacetyl derivatives. Excess reagent was removed under nitrogen and the dry residue was dissolved in $100 \mu$ l ethyl acetate.

Conditions of GCMS analysis. Analysis by GCMS was done using a Finnigan 4021 quadrupole mass spectrometer coupled with a direct transfer line to a Finnigan gas chromatograph and to the INCOS data system for data processing. The GC column was $2 \mathrm{~mm}$ ID by $180 \mathrm{~cm} \mathrm{3 \%} \mathrm{SP-2340} \mathrm{(Supelco,} \mathrm{Bellefonte,} \mathrm{PA),}$ and the column temperature was $220^{\circ} \mathrm{C}$ isothermal. Helium was the carrier gas, and the flow was $20 \mathrm{ml} / \mathrm{min}$. The injector temperature was $255^{\circ} \mathrm{C}$, the direct transfer line was $280^{\circ} \mathrm{C}$, and the ion source was $220^{\circ} \mathrm{C}$. Five $\mu \mathrm{l}$ of the samples or standards were injected, corresponding to $0.25 \mathrm{nmol}$ of $\mathrm{D}_{2}$-galactitol hexaacetate and 0.0125 to $25 \mathrm{nmol}$ of the hexitol hexaacetates. Similar results were obtained with the injection of 5 or $10 \mu \mathrm{l}$. Ammonia was used as the reagent gas for CI. The ammonia pressure in the source was 9 pascals, and the ionizing voltage was $70 \mathrm{eV}$. The following $\mathrm{CI}$ pseudomolecular ions, $\mathrm{M}^{+}+18\left(\mathrm{M}+\mathrm{NH}_{4}\right)^{+}$were chosen for selected ion monitoring: $\mathrm{m} / z 454$ for $\mathrm{D}_{2}$-galactitol hexaacetate, 452 for galactitol hexaacetate, mannitol hexaacetate, and sorbitol hexaacetate, and 450 for inositol hexaacetate. The dwell time was $100 \mathrm{msec}$ at each $\mathrm{m} / z$. Peak areas were calculated from the selected ion chromatograms using the computer after the operator visually selected the base line points.

Calibration curves were developed for galactitol, mannitol, sorbitol, and inositol. Aqueous standard solutions containing 5 $\mathrm{nmol}$ of $\mathrm{D}_{2}$-galactitol and variable amounts of galactitol $(0.25-$ $20 \mathrm{nmol})$, mannitol $(0.25-20 \mathrm{nmol})$, sorbitol (10-500 nmol), and inositol (10-500 $\mathrm{nmol}$ ) were prepared and carried through the identical procedure as the amniotic fluids. The peak areas at $\mathrm{m} / z 454$ for the $\mathrm{D}_{2}$-galactitol hexaacetate internal standard were corrected by subtraction of $5 \%$ of the area at $\mathrm{m} / \mathrm{z} 452$ for galactitol hexaacetate. This removed the contribution of the isotope cluster ion at $\mathrm{m} / z 454$ of galactitol hexaacetate. Calibration curves were plotted as the ratio of the SIM peak areas of the pseudomolecular ions of the compounds to the corrected SIM peak areas of the pseudomolecular ion of the internal standard $\mathrm{D}_{2}$-galactitol hexaacetate versus the molar ratios of the compounds to $\mathrm{D}_{2}$-galactitol and the lines were calculated by linear regression analysis. The amount of galactitol and the other hexitols in the amniotic fluid samples were calculated from the ratios of the SIM areas of the pseudomolecular ions of the compounds to the corrected SIM areas of the $\mathrm{D}_{2}$-galactitol hexaacetate internal standard using the equation of the standard calibration curve obtained for each compound.

The measurement of low levels of galactitol in amniotic fluid is complicated by the relatively large amounts of glucose present. On the 3\% SP-2340 GC column, the separation of the hexaacetates of mannitol, galactitol, sorbitol, and inositol is excellent, but glucose pentaacetate coelutes with galactitol hexaacetate. The glucose pentaacetate with a pseudomolecular ion of $\mathrm{m} / z 408$ has no interfering fragment or cluster ion which would confound the SIM analysis of the hexaacetates of galactitol, mannitol, sorbitol, or inositol; its high concentration causes a fluctuation of the ammonia pressure and therewith an instability of the SIM analysis of galactitol hexaacetate. It was for this reason that the Dowex 2-X8 $\left(\mathrm{OH}^{-}\right.$form) column was employed to retard glucose sufficiently to obtain in almost complete $(98 \%)$ separation from the faster eluting galactitol (2). The method was modified to collect not only the first but the second $3 \mathrm{ml}$ of eluate. Galactitol and inositol were not detectable in the second portion but $25 \%$ of the total mannitol and $5 \%$ of the total sorbitol were eluted with the second $3 \mathrm{ml}$. The recoveries of galactitol and $\mathrm{D}_{2-}$ galactitol were $80 \%$, which is comparable to the $84 \%$ reported by Allen et al. (2). Recovery of mannitol was nearly $75 \%$ and sorbitol $70 \%$, while the recovery of inositol was close to $100 \%$. Recoveries were assessed at concentrations between 1 and 100 nmol and did not vary with the actual concentration of these compounds. The recovery of $\mathrm{D}_{2}$-galactitol from the amniotic fluid samples was $80 \%$, similar to the recovery of $80 \%$ with the aqueous standards. Since galactitol and $\mathrm{D}_{2}$-galactitol had the same recoveries from aqueous standard solutions, the standard curves which are based on the ratios of galactitol to $D_{2}$-galactitol are valid for the amniotic fluid samples. Electron impact ionization of galactitol hexaacetate was found to produce a considerable fragmentation of the molecule. A pseudomolecular ion could not be seen, and in the upper mass range there were no abundant characteristic ions. The base peak was $\mathrm{m} / z 115$ which comprised only $10 \%$ of the total ions. For mannitol, sorbitol, and inositol hexaacetates, the situation was similar. Therefore, chemical ionization was chosen.

\section{RESULTS}

Figure 1 illustrates the CI mass spectra for galactitol hexaacetate and its deuterated analog. The relatively mild chemical ionization leads to a small amount of fragmentation and a mass spectrum containing a high abundance of the pseudomolecular ion of $(\mathbf{M}+18)^{+}$. The base peak $m / z 452$ for galactitol hexaacetate amounted to $55 \%$ of the total ions. A similar magnitude was found for $\mathrm{m} / \mathrm{z} 454$ in the case of $\mathrm{D}_{2}$-galactitol hexaacetate. SIM analyses of galactitol hexaacetate and the internal standard were therefore carried out at $\mathrm{m} / z 452$ and 454 , respectively. For galactitol hexaacetate, there is an isotope cluster at $\mathrm{m} / z 452,453$, and 454 , in which 454 comprises about $5 \%$ of the base peak at 

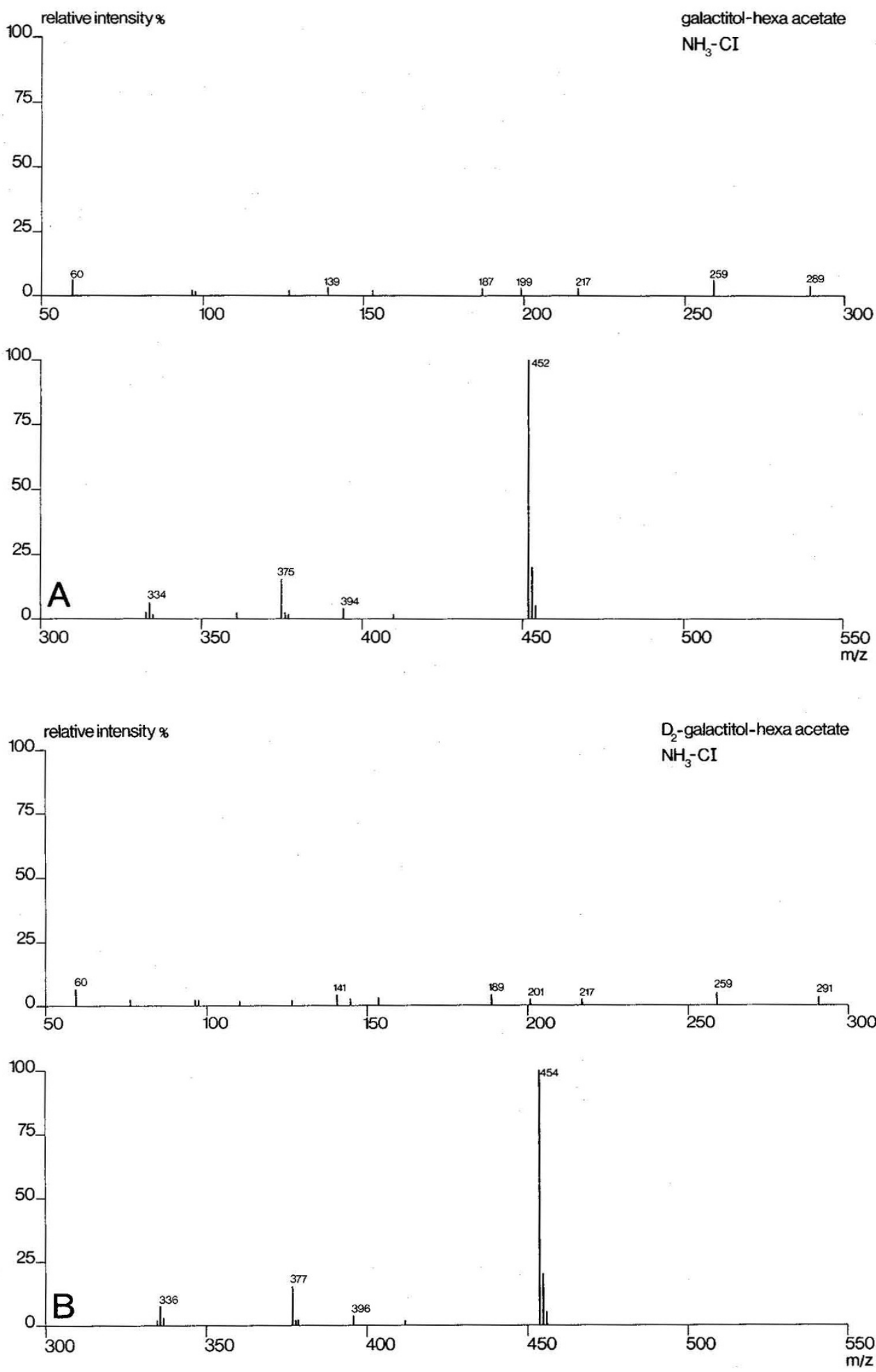

Fig. 1. A, Ammonia chemical ionization mass spectrum of galactitol hexaacetate. The base peak is at $m / z 452$; the pseudomolecular ion is (M + $18)^{+} . B$, Ammonia chemical ionization mass spectrum of $\mathrm{D}_{2}$-galactitol hexaacetate. The base peak is at $m / z 454$; the pseudomolecular ion is $(\mathrm{M}+$ $18)^{+}$.

$m / z$ 452. The same isotope cluster was found for mannitol and sorbitol hexaacetates and the base peaks at $\mathrm{m} / z 452$ were chosen for SIM. For inositol hexaacetate, the isotope cluster is $\mathrm{m} / z 450$, 451 , and 452 and the base peak at $\mathrm{m} / z 450$ was chosen for SIM. Figure 2 presents typical SIM chromatograms of a normal amniotic fluid. The SIM chromatograms at the $\mathrm{m} / \mathrm{z}$ of the pseudomolecular ions were not normalized in order to show the small peaks' of galactitol hexaacetate (peak $A$ ) at $m / z 452, \mathrm{D}_{2^{-}}$ galactitol hexaacetate (peak $B$ ) at $m / z 454$, and mannitol hexaa- cetate (peak $C$ ) at $\mathrm{m} / \mathrm{z} 452$ in the presence of the much larger peaks of sorbitol hexaacetate (peak $D$ ) at $\mathrm{m} / z 452$ and myoinositol hexaacetate (peak $E$ ) at $\mathrm{m} / z 450$. the relative intensities of the isotopic ions with an $\mathrm{m} / \mathrm{z}$ two higher than the pseudomolecular ions were about $5 \%$. With the large amounts of sorbitol hexaacetate, this gave a large peak at $\mathrm{m} / \mathrm{z} 454$ as well as $\mathrm{m} / \mathrm{z}$ 452. Similarly, the large amount of myoinositol hexaacetate gave a large peak at $\mathrm{m} / z 452$ as well as at $\mathrm{m} / z 450$. The concentration of galactitol in this amniotic fluid sample was $0.91 \mu \mathrm{mol} / \mathrm{liter}$. 


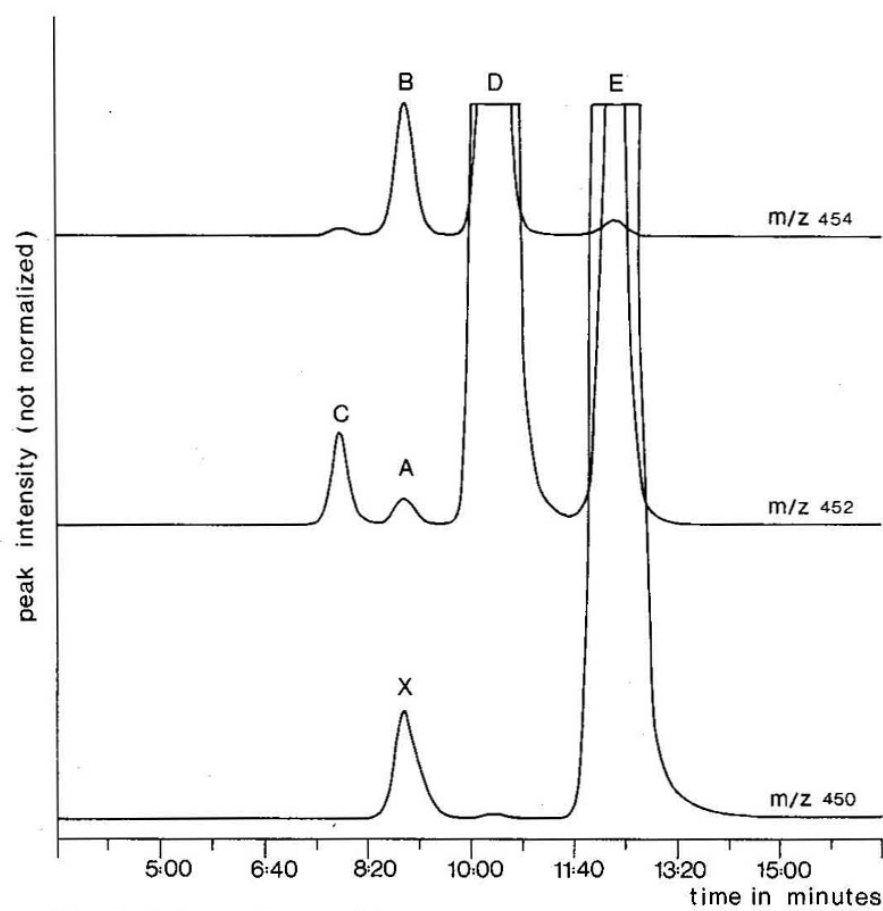

Fig. 2. Selected ion monitoring ammonia chemical ionization gas chromatograms of the hexaacetate derivatives from normal amniotic fluid. Peak $A$, galactitol hexaacetate pseudomolecular ion at $\mathrm{m} / \mathrm{z} 452$; peak $B, \mathrm{D}_{2}$-galactitol hexaacetate pseudomolecular ion at $\mathrm{m} / \mathrm{z} 454$; peak $C$, mannitol hexaacetate pseudomolecular ion at $\mathrm{m} / \mathrm{z} 452$; peak $D$, sorbitol hexaacetate pseudomolecular ion at $m / z 452$ and isotopic ion at $\mathrm{m} / \mathrm{z} 454$; peak $E$, myoinositol hexaacetate pseudomolecular ion at $\mathrm{m} / \mathrm{z}$ 450 and isotopic ion at $m / z 452$; and peak $X$, unknown ion at $m / z 450$.

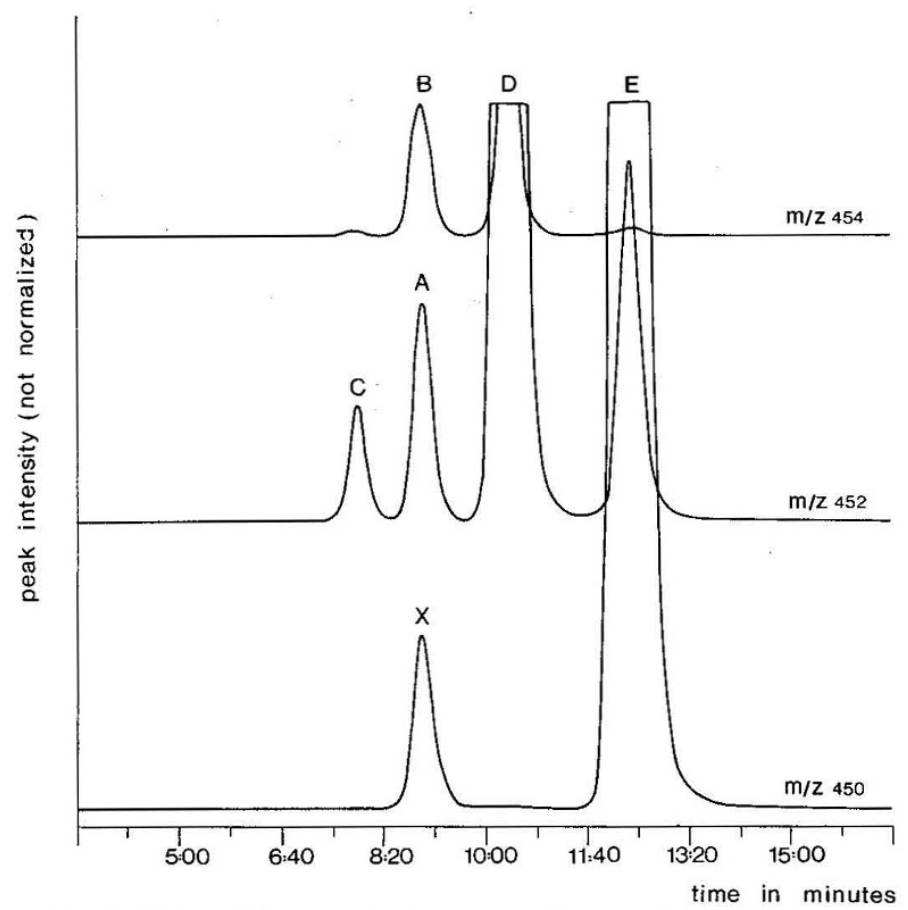

Fig. 3. Selected ion monitoring ammonia chemical ionization gas chromatograms of the hexaacetate derivatives from amniotic fluid of a fetus with galactosemia. Peak A, galactitol hexaacetate pseudomolecular ion at $m / z 452$; peak $B, \mathrm{D}_{2}$-galactitol hexaacetate pseudomolecular ion at $m / z 454$ with a $6.8 \%$ contribution of the isotopic ion of galactitol hexaacetate at $\mathrm{m} / z$ 454; peak $C$, mannitol hexaacetate pseudomolecular ion at $m / z 452$; peak $D$, sorbitol hexaacetate pseudomolecular ion at $m /$ $z 452$ and isotopic ion at $\mathrm{m} / z$ 454; peak $E$, myoinositol hexaacetate pseudomolecular ion at $\mathrm{m} / z 450$ and isotopic ion at $\mathrm{m} / \mathrm{z} 452$; and peak $X$, unknown ion at $m / z 450$.
In Figure 3, the SIM chromatograms are those of the amniotic fluid of a pregnant woman carrying a fetus affected with galactosemia. The concentration of galactitol in this amniotic fluid sample was $7.96 \mu \mathrm{mol} /$ liter. The distinct positions of the hexaacetates of galactitol ( peak A), mannitol (peak $C$ ), sorbitol (peak $D)$, and inositol (peak $E$ ) are shown in Figures 2 and 3. In addition, there was an unknown peak (peak $X$ ) on the SIM trace of $m / z 450$, eluting with galactitol hexaacetate. Because of the isotope cluster, there is a large contribution from sorbitol hexaacetate to the SIM trace of $\mathrm{m} / \mathrm{z} 454$ and from inositol hexaacetate to the SIM trace of $\mathrm{m} / z$ 452. There is also a contribution of approximately $5 \%$ from galactitol hexaacetate to the area of $\mathrm{D}_{2}$ galactitol hexaacetate, which is corrected for this contribution.

In the standard calibration curve (Fig. 4) there was good linearity of the ratio of the areas at $\mathrm{m} / \mathrm{z} 452$ to 454 (corrected for the contribution of galactitol) versus the ratio of the variable amounts of galactitol $(0.25-20 \mathrm{nmol})$ to the constant amount of $\mathrm{D}_{2}$-galactitol $(5 \mathrm{nmol}$ ). The correlation coefficient was $>0.999$. The calibration curve for mannitol using $\mathrm{D}_{2}$-galactitol as internal standard also displayed very good linearity with a correlation coefficient of $>0.999$. The calibration curves for sorbitol and inositol were satisfactorily linear in the lower region $(0.25-20$ $\mathrm{nmol})$, but the extended curves $(10-500 \mathrm{nmol})$ were less linear, and therefore the amounts in amniotic fluid calculated from the calibration curves were less accurate.

The concentrations of galactitol, mannitol, sorbitol, and inositol found in normal amniotic fluid and that surrounding a fetus with galactosemia are summarized in Table 1 . The mean normal concentration of galactitol was $0.70 \pm 0.18$ (1 SD) $\mu \mathrm{mol} / \mathrm{liter}$. The range was 0.44 to $0.91 \mu \mathrm{mol} / \mathrm{liter}$. The level of galactitol in the amniotic fluid of the fetus with galactosemia was $7.96 \mu \mathrm{mol} /$

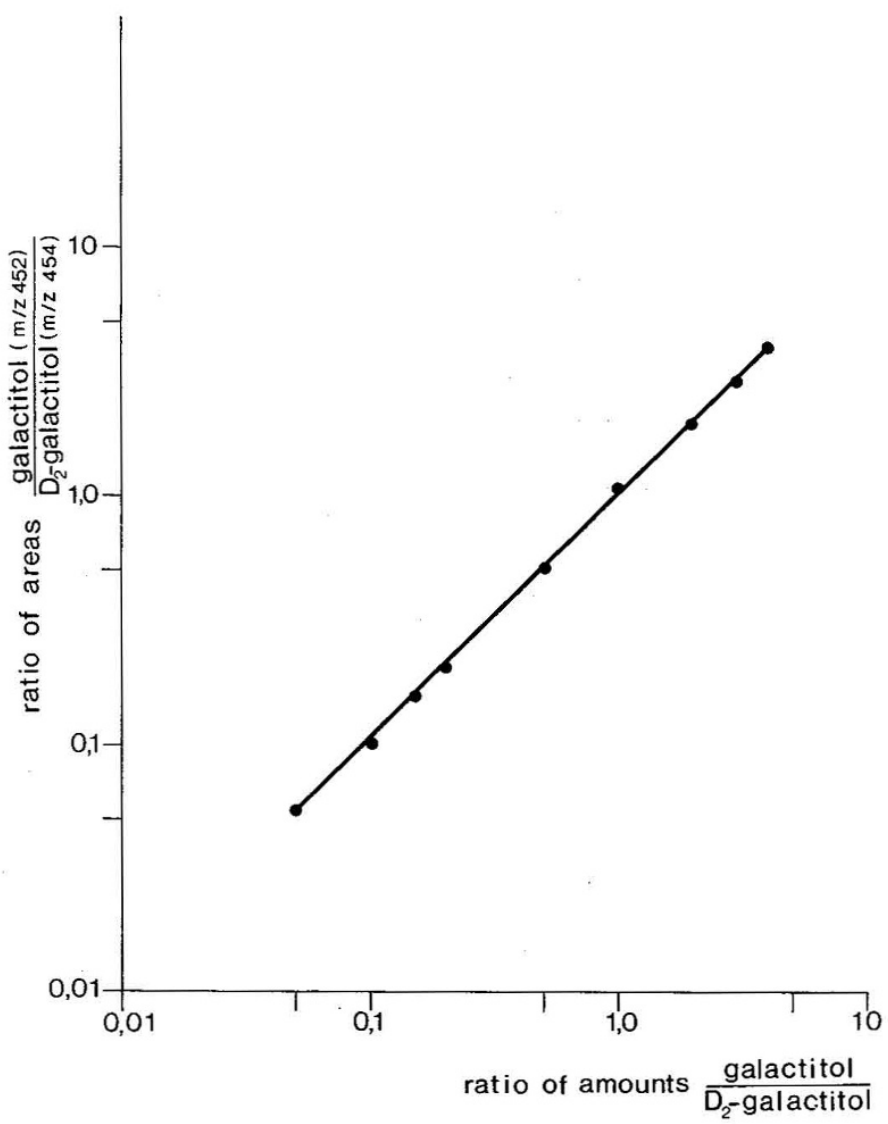

Fig. 4. Standard curve for selected ion monitoring ammonia chemical ionization gas chromatography-mass spectrometry of galactitol. The ratios of peak areas for galactitol hexaacetate $(\mathrm{m} / z 452)$ to the peak areas of the internal standard, $\mathrm{D}_{2}$-galactitol hexaacetate $(\mathrm{m} / \mathrm{z} 454)$ (corrected for the isotopic contribution of galactitol hexaacetate) are plotted against the mole ratio of galactitol to the internal standard $\mathrm{D}_{2}$-galactitol $(5 \mathrm{nmol})$. 
Table 1. Concentrations of galactitol, mannitol, sorbitol, and inositol in amniotic fluid of controls and a fetus with galactosemia.

\begin{tabular}{|c|c|c|c|c|c|}
\hline \multirow[b]{2}{*}{ Gestational age } & \multirow[b]{2}{*}{ Subject } & \multicolumn{4}{|c|}{ Concentration $(\mu \mathrm{Mol} / \mathrm{I})$} \\
\hline & & Galactitol & Mannitol & Sorbitol & Inositol \\
\hline & Normal 1 & 0.80 & 3.24 & 102.90 & 79.47 \\
\hline & Normal 2 & 0.91 & 4. 18 & 176.09 & 63.12 \\
\hline & Normal 3 & 0.72 & 1.84 & 90.26 & 43.83 \\
\hline & Normal 4 & 0.44 & 8.92 & 64.32 & 15.19 \\
\hline & Normal 5 & 0.64 & 1.73 & 188.55 & 92.50 \\
\hline $16-18$ weeks & $(n=5) \quad \bar{x}=$ & $0.70 \pm 0.18$ & $3.78 \pm 2.94$ & $124.41 \pm 54.41$ & $58.83 \pm 30.40$ \\
\hline
\end{tabular}

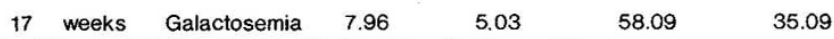

liter, which was 10 times the control mean and 40 SD above the normal mean. Mannitol was found to be a normal constituent of amniotic fluid. Data are also provided on the broad range of concentrations of sorbitol and inositol in amniotic fluid.

\section{DISCUSSION}

Stable isotope dilution assay for galactitol in amniotic fluid provides a rapid, sensitive, and accurate method for prenatal diagnosis. The deuterium-labeled galactitol serves as a carrier during preparation of the sample for GCMS, and measurement of the ratio of nonlabeled to labeled compound corrects for any losses during the ion exchange, derivatization and GC procedures. Measurement of the internal standard verifies that the preparation of the sample was satisfactory and enhances confidence in the results. The use of $\mathrm{D}_{2}$-galactitol, a nonphysiological compound, as internal standard is clearly preferable to use of mannitol, which has been used in reported procedures $(2,3,9)$ but has been criticized because this compound is present endogenously (8).

The use of $\mathrm{D}_{2}$-galactitol as an internal standard has also permitted us to obtain accurate quantitative data on the concentration of mannitol in amniotic fluid and semiquantitative data on the concentrations of sorbitol and inositol. The coeluting compound which has a retention time close to that of galactitol hexaacetate on the SIM trace of $\mathrm{m} / z 450$ would not be distinguished in a simple GC analysis. This might be the explanation for the 2-fold higher normal value of $1.40 \mu \mathrm{mol} /$ liter for galactitol in amniotic fluid reported by Allen et al. (2) compared to the value of $0.70 \mu \mathrm{mol} / \mathrm{liter}$ which has been found by stable isotope dilution. This compound has not been identified. It could be an isomer of inositol. As many as nine isomers of inositol are known of which myoinositol is the major compound occurring physiologically.
The measurement of galactitol in amniotic fluid by stable isotope dilution should avoid the need for culturing amniotic fluid cells. This is not only time consuming, but cells may fail to grow, or contamination and overgrowth by cells of maternal origin may give a false negative result (4). Nevertheless, until more experience is gained, it would be prudent to monitor diagnosis by direct analysis of galactitol with analysis of enzyme activity in cultured cells. With experience, it should be practical to utilize this method alone. The method could be extended to any other body fluid in which the measurement of a very low concentration of galactitol is of interest. The possibility that the measurement of concentrations of galactitol in maternal blood or urine could provide for prenatal diagnosis of galactosemia has not been explored.

\section{REFERENCES AND NOTES}

1. Allen JT, Gillett M, Holton JB, King GS, Pettit B 1980 Evidence of galactosemia in utero. Lancet 1:603

2. Allen JT, Holton JB, Gillett MG 1981 Gas-liquid chromatographic determination of galactitol in amniotic fluid for possible use in prenatal diagnosis of galactosemia. Clin Chim Acta 110:59

3. Batti T, Clamp JR 1968 Identification and estimation of monosaccharides and disaccharides in urine by gas-luqiid chromatography. Clin Chim Acta 22:563

4. Buchanan PD, Kahler SC, Sweetman L, Nyhan WL 1980 Pitfalls in the prenata diagnosis of propionic acidemia. Clin Genet 18:177

5. Fensom AH, Benson PF 1975 Assay of galactose-1-phosphate uridyl transferase in cultured amniotic fluid cells for prenatal diagnosis of galactosemia. Clin Chim Acta 62:189

6. Gitzelmann R, Hanson RG 1980 Galactose metabolism, hereditary defects and their clinical significance. In: Burman D, Holton JB, Pennock CA (eds): Inherited Disorders of Carbohydrate Metabolism, ed 1. Lancaster, MTP Press, p 61

7. Holton JB, Gillett MG, McFaul R, Yound R 1981 Galactosemia: a new severe variant due to uridine diphosphate galactose-4-epimerase deficiency. Arch Dis Child 56:885

8. Lake MF, Gunn WG 1979 Natural occurrence disqualifies mannitol as an internal standard when urinary monosaccharides are determined by gasliquid chromatography. Clin Chim Acta 96:265

9. Murphy D, Pennock, CA 1972 Gas chromatographic measurement of blood and urine glucose and other monosaccharides. Clin Chim Acta 42:67

10. Ng, WG, Donnell NG, Bergren WR, Alfi O, Golbus MS 1977 Prenatal diagnosis of galactosemia. Clin Chim Acta 74:227

11. Roe TF, Hallatt JG, Donnell GN, Ng WG 1971 Childbearing by a galactosemic women. J Pediatr 78:1026

12. Segal S 1978 Disorders of galactose metabolism. In: Stanbury JB, Wyngaarden JB, Fredrickson DS (eds): The Metabolic Basis of Inherited Disease, ed 4 New York, McGraw-Hill, p 160

13. Tedesco TA, Morrow G, Mellman WJ 1972 Normal pregnancy and childbirth in a galactosemic woman. J Pediatr 81:1159

14. This research was supported in part by United States Public health Services Grant HD 04608 from the National institute of Child Health and Human Development and Grants GM-17702 and NS-19213 from the National Institute of General Medical Sciences, National Institutes of Health.

15. The authors express appreciation to Dr. O. W. Jones (San Diego) for providing normal amniotic fluids and to Dr. M. F. Niermeyer (Rotterdam) for providing the amniotic fluid of the fetus with galactosemia.

16. On leave from the Department of Pediatrics, Free University of Amsterdam De Boelelaan 1117, 1007 MB Amsterdam, The Netherlands. Reprint requests should be addressed here.

17. Received for publication June 25,1983

18. Accepted for publication February 12, 1984. 